\title{
Uma Arquitetura para Sistemas de Saúde Eletrônicos que Suporta o Monitoramento de Pacientes e a Notificação de Cuidadores com base em Raciocínio Automático para evitar a Fadiga de Alarme
}

\author{
Chrystinne Oliveira Fernandes ${ }^{1}$, Carlos José Pereira de Lucena ${ }^{2}$ \\ ${ }^{1}$ Departamento de Informática - Pontifícia Universidade Católica do Rio de Janeiro \\ (PUC-Rio) \\ Caixa Postal 22452-900 - Rio de Janeiro - RJ - Brasil \\ chrystinne@gmail.com, lucena@inf.puc-rio.br
}

\begin{abstract}
Resumo. Estimativas informam que $80 \%$ a 99\% dos alarmes disparados em unidades hospitalares são falsos ou clinicamente insignificantes, não apresentando perigo real aos pacientes. Estes falsos alertas podem culminar em uma sobrecarga de alertas que leva um profissional da saúde a perder eventos importantes que podem ser prejudiciais aos pacientes ou até mesmo fatais. À medida que as unidades de saúde se tornam mais dependentes de dispositivos de monitoramento que acionam alarmes, o problema da fadiga de alarme deve ser tratado como uma das principais questões, a fim de prevenir a sobrecarga de alarme para os profissionais da saúde e aumentar a segurança do paciente. O principal objetivo desta tese é propor uma solução para o problema de fadiga de alarme usando um mecanismo de raciocínio automático para decidir como notificar os membros da equipe de saúde. Esta tese descreve: um modelo para suportar algoritmos de raciocínio que decidem como notificar os profissionais de saúde para evitar a fadiga de alarme; uma arquitetura para sistemas de saúde que suporta recursos de monitoramento, raciocínio e notificação de pacientes; e três algoritmos de raciocínio que decidem: (i) como notificar os profissionais de saúde decidindo quando agrupar um conjunto de alarmes; (ii) se deve ou não notificar os profissionais de saúde com uma indicação de probabilidade de falso alarme; (iii) quem é o melhor cuidador a ser notificado considerando um grupo de cuidadores.
\end{abstract}

\section{Introdução}

A Tecnologia da Informação (TI) já tem provido benefícios significativos para o setor da Saúde, mas ainda existem muitas áreas onde a aplicação da TI poderia oferecer melhorias críticas adicionais. Por exemplo, um problema hospitalar mundial hoje em dia é a Fadiga do Alarme (FA) (Hussain et al., 2019), que recentemente vem recebendo atenção da Indústria, do setor de Saúde e da comunidade acadêmica.

A FA envolve uma falta de resposta devido ao número excessivo de alarmes não-críticos recebidos pela equipe de cuidadores da saúde, resultando em uma sobrecarga sensorial para o staff (Cvach, 2012). Para ilustrar a severidade deste problema, no Canadá e nos Estados Unidos, onde esta questão tem sido tratada como uma grande preocupação (Jones, 2014), foi reportado o resultado da análise do sistema 
de alarmes do hospital The Johns Hopkins, em Baltimore, ocorrido durante um período de 12 dias. $\mathrm{O}$ estudo mostrou que houve uma média de 350 alertas por leito por dia. Em uma Unidade de Tratamento Intensivo (UTI), os números foram ainda mais expressivos, com uma média de 771 alertas sendo disparados por leito por dia. Tais números indicam uma sobrecarga sensorial grave para o staff médico, com sérias consequências para a segurança dos pacientes se um alarme for ignorado.

Neste trabalho, apresentamos uma nova abordagem para lidar com o problema da FA, suas causas mais comuns e consequências adversas. Nossa solução utiliza uma abordagem de inteligência artificial baseada em um sistema de raciocínio automático que decide como notificar cuidadores da saúde sobre anomalias detectadas por um sistema de monitoramento de pacientes onde um grande volume de alarmes pode levar à FA. Com isso, visamos avaliar a validade dos alarmes e notificar, dentre os cuidadores, o membro mais apropriado para receber alarmes que realmente requerem atenção.

\subsection{Definição do Problema}

Uma preocupação em um grande número de hospitais e uma queixa entre possivelmente todo time médico que trabalha com monitores fisiológicos, a Fadiga do Alarme é causada pelo excessivo número de alarmes gerados pelos dispositivos de monitoramento. A FA representa uma questão substancial que pode trazer consequências sérias para o atendimento à saúde, podendo culminar em um ambiente inseguro para o paciente. Por exemplo, podemos citar a dessensibilização do time médico em relação aos alertas, o que pode levar a longos tempos de resposta para tratar as anomalias assim como a negligência de eventos críticos.

\subsection{Motivação e Objetivos}

Estimativas mostram que $80 \%$ a $99 \%$ dos alarmes disparados em unidades hospitalares são falsos ou clinicamente insignificantes. Tais alarmes representam uma cacofonia de sons que sequer representam um perigo real para os pacientes. Consequentemente, estes falsos alarmes podem levar a uma sobrecarga de alarmes que pode comprometer a atenção dos cuidadores de saúde (Cvach, 2012; Drew et al., 2014; Jones, 2014; Tanner, 2013), podendo levá-los a perder alarmes relevantes que anunciam eventos significativos ou que representam risco de vida aos pacientes. A medida que as unidades de atendimento à saúde se tornam mais dependentes de dispositivos de monitoramento de sinais vitais utilizados no cuidado com os pacientes, a questão da FA deve ser tratada com a devida importância. Neste caso, estratégias factíveis precisam ser providas para prevenir a sobrecarga de alarmes e aumentar a segurança do paciente.

Nosso principal objetivo neste trabalho é propor uma solução que contribua para a mitigação do problema da FA. A solução proposta se baseia em um mecanismo de raciocínio que decide como notificar cuidadores da saúde, indicando, por exemplo, uma Probabilidade de Falso Alarme (PFA). O rótulo indicativo de PFA adicionado à notificação pode auxiliar os cuidadores a priorizar seus trabalhos, especialmente quando eles estão sob condições de fadiga do alarme. Também visamos reduzir a sobrecarga de alarmes gerados pelos monitores nos leitos através do agrupamento de notificações semelhantes. Finalmente, também objetivamos raciocinar sobre qual cuidador é o mais apropriado para receber a notificação, considerando o grau de experiência, disponibilidade, geolocalização e as circunstâncias da carga de trabalho do staff. 


\section{Resultados}

\subsection{Raciocinando sobre Como Notificar para evitar a Fadiga do Alarme}

Nesta seção, discutimos nosso algoritmo desenvolvido para mitigar a FA através do uso de um mecanismo de raciocínio que notifica os cuidadores agrupando alertas semelhantes (Fernandes et al., 2019). A solução que fornecemos permite reduzir o número de notificações recebidas pelo staff médico, de modo que eles possam focar nas atividades que realmente requerem a devida atenção. Nossos experimentos foram configurados para alertar enfermeiros e foram avaliados através do uso de um dataset de dados reais de pacientes submetidos à anestesia e em monitoramento durante 32 casos cirúrgicos, no hospital Royal Adelaide (Liu, Görges, \& Jenkins, 2012). Este dataset foi escolhido por fornecer os dados de sinais vitais - batimento cardíaco - necessários à realização dos nossos experimentos, de acordo com o seu projeto.

Nosso principal objetivo neste estudo de caso foi determinar como agrupar um conjunto de alarmes ocorridos em um curto intervalo de tempo (chamado de MNI) para entregá-los juntos. Nosso objetivo específico foi evitar que alarmes do mesmo tipo, e que foram disparados para o mesmo paciente, fossem alertados mais de uma vez dentro do MNI, adotando-se uma estratégia de atraso na entrega de uma dada notificação. Neste estudo de caso, definimos um MNI de 5 minutos para todos os experimentos. Os delays ocorridos entre o disparo dos alertas e a entrega das notificações, para o experimento 5, podem ser verificados através da visualização dos gráficos plotados em (Fernandes et al., 2019). Ao final dos experimentos, comparamos o número de alarmes disparados em nosso sistema com o número de notificações entregues aos cuidadores. Nossos resultados mostraram que a redução no total de notificações recebidas pelos cuidadores foi de até 99.3\% (582/586) do total de alarmes, com uma média de 99.17\% (1912/1929) de redução no número total de alarmes, considerando todos os experimentos.

\subsection{Raciocinando sobre como detectar falsos alarmes através da análise de informação de contexto dos alarmes}

Nesta seção, nós discutimos como podemos raciocinar sobre como adicionar uma indicação de probabilidade de falso alarme a uma notificação (FAP_LABEL) (Fernandes et al., 2019). O FAP_LABEL é calculado de acordo com indicadores de falso alarme que nós definimos com base na nossa revisão da literatura. A ideia é utilizar estes indicadores para decidir quando uma anomalia reportada através de uma notificação poderia ser analisada pela equipe de cuidadores com um nível mais baixo de prioridade. Neste caso, o FAP_LABEL poderia ser utilizado para auxiliar os cuidadores a priorizarem o próximo alarme a ser atendido.

O mecanismo de raciocínio associa uma PFA aos alarmes com base em informações de contexto dos alarmes coletadas em tempo real, incluindo: (i) sensores e (ii) circunstâncias dos pacientes, como seu posicionamento na cama e sua localização (rastreada pelo uso de dispositivos wearables com GPS (Global Positioning System)). Depois de receber tais informações de contexto, o algoritmo de raciocínio inicia a análise de cada alarme e calcula o PFA associado a ele de acordo com os indicadores de falso alarme. Então, o sistema de raciocínio utiliza a PFA calculada para cada alarme 
para decidir quando incluir uma indicação de probabilidade de falso alarme (FAP_LABEL) para uma notificação que poderá ser visualizada pelos cuidadores.

Os quatro indicadores de falsos alertas definidos neste estudo de caso representam informações sobre (i) a duração do nível de bateria de um determinado sensor e a última vez que ela foi trocada, (ii) a última vez que a pele do paciente foi preparada para receber eletrodos e a última vez que eles foram trocados, (iii) o deslocamento do paciente, e (iv) a posição do paciente na cama. Para calcular o percentual de indicação de falso alarme em nosso experimento, nós consideramos que cada indicador tem o mesmo peso.

\subsection{Raciocinando sobre Quem notificar}

O nosso algoritmo de mitigação da FA que decide qual o melhor cuidador a ser notificado, dentre o time de cuidadores, analisa as seguintes informações da UTI: (i) contexto do alarme, (ii) condições dos pacientes (por exemplo, seu grau de severidade), e (iii) circunstâncias dos cuidadores (nível de experiência, distância física até o paciente que precisa de cuidados e quantas notificações eles já receberam anteriormente).

Nossa estratégia para decidir a melhor atribuição $e$ para cada notificação é calcular a função benefício benefit(e) para cada possível atribuição $e$, onde as possíveis atribuições são aquelas que respeitam as seguintes restrições: 1- um cuidador deve apenas receber notificações de acordo com sua capacidade; 2- um cuidador pode apenas receber uma notificação $n$ se ele/ela estiver disponível no momento em que a notificação ocorre; 3- uma notificação deve ser preferencialmente atribuída ao cuidador que possui mais experiência; 4- uma notificação deve ser preferencialmente atribuída ao cuidador que estiver fisicamente mais próximo do paciente; 5- uma notificação deve ser preferencialmente atribuída aos cuidadores que tiverem recebido menos notificações.

A implementação calcula o valor correspondente ao benefício de cada possível atribuição (de uma notificação a um cuidador) e decide a melhor opção dentre todos os benefícios calculados, através de uma estratégia gulosa.

\subsection{Discussão}

Experimentos foram realizados para demonstrar que, ao fornecer um sistema de raciocínio que agrupa alarmes semelhantes e recorrentes, pode-se reduzir o total de notificações recebidas pelos cuidadores em até $99.3 \%$ do total de alarmes gerados. Esses experimentos foram avaliados através do uso de dados reais de monitoramento de sinais vitais de pacientes registrados durante 32 casos cirúrgicos nos quais os pacientes foram submetidos à anestesia, no hospital Royal Adelaide. Apresentamos os resultados desse algoritmo através de gráficos gerados na linguagem $\mathrm{R}$, onde mostramos se o algoritmo decidiu emitir um alarme imediatamente ou após um determinado delay.

Também mostramos que nosso mecanismo de raciocínio foi capaz de indicar a probabilidade de um alarme ser falso com base em indicadores de falsos alarmes. Nossos experimentos foram conduzidos para demonstrar que podemos raciocinar sobre como detectar falsos alarmes através da análise de informações de contexto dos alarmes.

Para a tarefa de atribuição de notificações realizada pelo nosso algoritmo de raciocínio que decide sobre qual cuidador notificar, também alcançamos nossos 
resultados esperados, uma vez que o algoritmo priorizou o cuidador que estava disponível no momento do alarme, além de ser o mais experiente e capaz de atender à notificação. Nós definimos os seguintes critérios de priorização: experiência do cuidador, probabilidade de um alarme ser falso, gravidade do paciente, distância física entre cuidadores e pacientes, e o número de notificações que os cuidadores já tinham recebido até o momento. Os resultados de nossos experimentos mostraram que, em nosso ambiente simulado, cuidadores com níveis de experiência mais altos receberam mais notificações que aqueles com níveis mais baixos de experiência.

\section{Conclusões}

A segurança do alarme é um problema complexo, influenciado por diversos fatores que extrapolam os desafios da tecnologia, como a influência de fatores humanos, condições dos pacientes e até questões culturais do staff (Keller, 2012). A medida que a prática de cuidados médicos torna-se mais orientada à tecnologia, estratégias inteligentes devem ser adotadas para que o staff responda aos chamados garantindo a segurança do paciente em um ambiente cada vez mais complexo (Tanner, 2013). Este trabalho propõe uma nova abordagem para lidar com o problema da FA, utilizando um mecanismo de raciocínio para decidir como notificar cuidadores sobre anomalias detectadas por um sistema de monitoramento onde um grande número de alarmes pode levar à FA.

Nós demonstramos através de nossos experimentos que prover um sistema de raciocínio pode reduzir o total de notificações recebidas pelos cuidadores em até $99.3 \%$ do total de alarmes gerados. De acordo com Winters et al., quase todos os estudos assumem que uma redução no número total de alarmes e/ou de falsos alarmes irá reduzir o problema da fadiga do alarme (Winters et al., 2018). Sendo assim, esperamos que nosso algoritmo possa ser utilizado como uma estratégia viável para mitigar a FA. Também esperamos que a nossa abordagem possa ser útil para auxiliar na prevenção das consequências negativas da FA, tais como: ruptura do cuidado com o paciente, desligamento dos sistemas de alarmes pelo staff, falta ou redução no tempo de resposta às anomalias, menor atuação em eventos reais, perda de eventos críticos, dentre outros.

\subsection{Principais Contribuições}

Neste trabalho, nós tentamos preencher o gap de ter soluções factíveis para mitigar o problema da FA, com enfoque na questão dos alarmes excessivos e dos falsos positivos que são reconhecidos mundialmente como problemas sérios e que ainda continuam sem solução. Através do uso de um processo de monitoramento e notificação inteligente suportado por um mecanismo de raciocínio que auxilia principalmente os cuidadores da saúde, nosso trabalho oferece principalmente as seguintes contribuições: 1- Uma arquitetura para sistemas de saúde que suporta o monitoramento de pacientes, raciocínio e notificação; 2- Um modelo de notificações que suporta algoritmos de raciocínio que decide como notificar cuidadores para evitar a FA sem comprometer a segurança do paciente; 3- Um algoritmo de raciocínio que especifica como notificar cuidadores através do agrupamento de alarmes semelhantes; 4- Um algoritmo de raciocínio que especifica como detectar falsos alarmes e notificar cuidadores com uma indicação de probabilidade de um alarme ser falso; 5- Um algoritmo de raciocínio que decide quem é o melhor cuidador a ser notificado dentre o time de cuidadores disponíveis em uma UTI. 


\subsection{Trabalhos Futuros}

Como plano futuro, visamos desenvolver uma versão otimizada do nosso algoritmo de raciocínio que calcula o indicativo de PFA baseado no volume de alarmes disparados em uma UTI em tempo real. A ideia é prover um sistema de classificação confiável que os cuidadores possam utilizar para auxiliá-los na priorização de seu atendimento, especialmente quando estiverem em condições de FA.

Outro trabalho futuro é evoluir o algoritmo de raciocínio sobre quem notificar para lidar com negociação por recursos, de modo que, se todos os cuidadores estiverem indisponíveis, seja possível solicitar que membros de outras equipes possam ser alocados aos times que já atingiram sua capacidade de responder aos alarmes.

Por fim, vislumbramos a realização de um estudo qualitativo para avaliar, com base nos estudos obtidos, como, por exemplo, a redução no número de alertas impacta no cuidado com o paciente.

Embora tenhamos avaliado nossos algoritmos em um ambiente experimental, tentamos reproduzir o contexto de um ambiente clínico utilizando dados reais de pacientes. Como trabalho futuro, visamos avaliar os resultados de nossos algoritmos utilizando condições clínicas mais realistas.

\section{Referências}

Hussain, Mustafa I. Reynolds, Tera L. Zheng, Kai. (2019). Medication safety alert fatigue may be reduced via interaction design and clinical role tailoring: a systematic review, Journal of the American Medical Informatics Association, Volume 26, Issue 10, October, Pages 1141-1149, https://doi.org/10.1093/jamia/ocz095

Cvach, M. (2012). Monitor Alarm Fatigue: An Integrative Review. Biomedical Instrumentation \& Technology, 46(4), 268-277. https://doi.org/10.2345/0899-820546.4.268

Jones, K. (2014). Alarm fatigue a top patient safety hazard. CMAJ : Canadian Medical Association Journal = Journal de 1'Association Medicale Canadienne, 186(3), 178. https://doi.org/10.1503/cmaj.109-4696

Drew, B. J., Harris, P., Zègre-Hemsey, J. K., Mammone, T., Schindler, D., Salas-Boni, R., ... Hu, X. (2014). Insights into the problem of alarm fatigue with physiologic monitor devices: a comprehensive observational study of consecutive intensive care unit patients. PloS One, 9(10), e110274. https://doi.org/10.1371/journal.pone.0110274

Tanner, T. (2013). The Problem of Alarm Fatigue. Nursing for Women's Health, 17(2), 153-157. https://doi.org/https://doi.org/10.1111/1751-486X.12025

Fernandes, Chrystinne, Miles, Simon, Cowan, Donald, \& Lucena, C.J.P. de. (2019). Artificial Intelligence Technologies for Coping with Alarm Fatigue in Hospital Environments Because of Sensory Overload. (JMIR - Journal of Medical Internet Research - ISSN 1438-8871)

Liu, D., Görges, M., \& Jenkins, S. A. (2012). University of Queensland Vital Signs Dataset: Development of an Accessible Repository of Anesthesia Patient Monitoring Data for Research. Anesthesia \& Analgesia, 114(3). Retrieved from https://journals.lww.com/anesthesiaanalgesia/Fulltext/2012/03000/University_of_Queensland_Vital_Signs_Dataset_.15 .aspx 
Winters, B. D., Cvach, M. M., Bonafide, C. P., Hu, X., Konkani, A., O’Connor, M. F., (2018). The Society for Critical Care Medicine Alarm and Alert Fatigue Task Force.. Technological Distractions (Part 2): A Summary of Approaches to Manage Clinical Alarms With Intent to Reduce Alarm Fatigue. Critical Care Medicine, 46(1). Retrieved from https://journals.lww.com/ccmjournal/Fulltext/2018/01000/Technological_Distraction s_Part_2_A_Summary_of.17.aspx

Fernandes, Chrystinne, Miles, Simon, Lucena, C.J.P. de. (2020). Reasoning about How to Detect False Alarms by Analyzing Alarm-context Information. Journal JMIR Medical Informatics - ISSN 2291-9694.

Keller, J. P. (2012). Clinical alarm hazards: a "top ten" health technology safety concern. Journal of Electrocardiology, 45(6), 588-591. https://doi.org/https://doi.org/10.1016/j.jelectrocard.2012.08.050 